\title{
Correction to: Circadian neurogenetics of mood disorders
}

Jorge Mendoza ${ }^{1} \cdot$ Guillaume Vanotti $^{1}$

Published online: 8 June 2019

(C) Springer-Verlag GmbH Germany, part of Springer Nature 2019

\section{Correction to: Cell and Tissue Research https://doi.org/10.1007/s00441-019-03033-7}

The published online version contains a mistake. Figures 1 and 2 of the final PDF were not changed according to author's request during the proof corrections.

The original article has been corrected.

The online version of the original article can be found at https://doi.org/ 10.1007/s00441-019-03033-7

Jorge Mendoza

jmendoza@inci-cnrs.unistra.fr

1 Institute of Cellular and Integrative Neurosciences, CNRS

UPR-3212 University of Strasbourg, 8 allée du Général Rouvillois, 67000 Strasbourg, France 
The correct Figures 1 and 2 are given below:

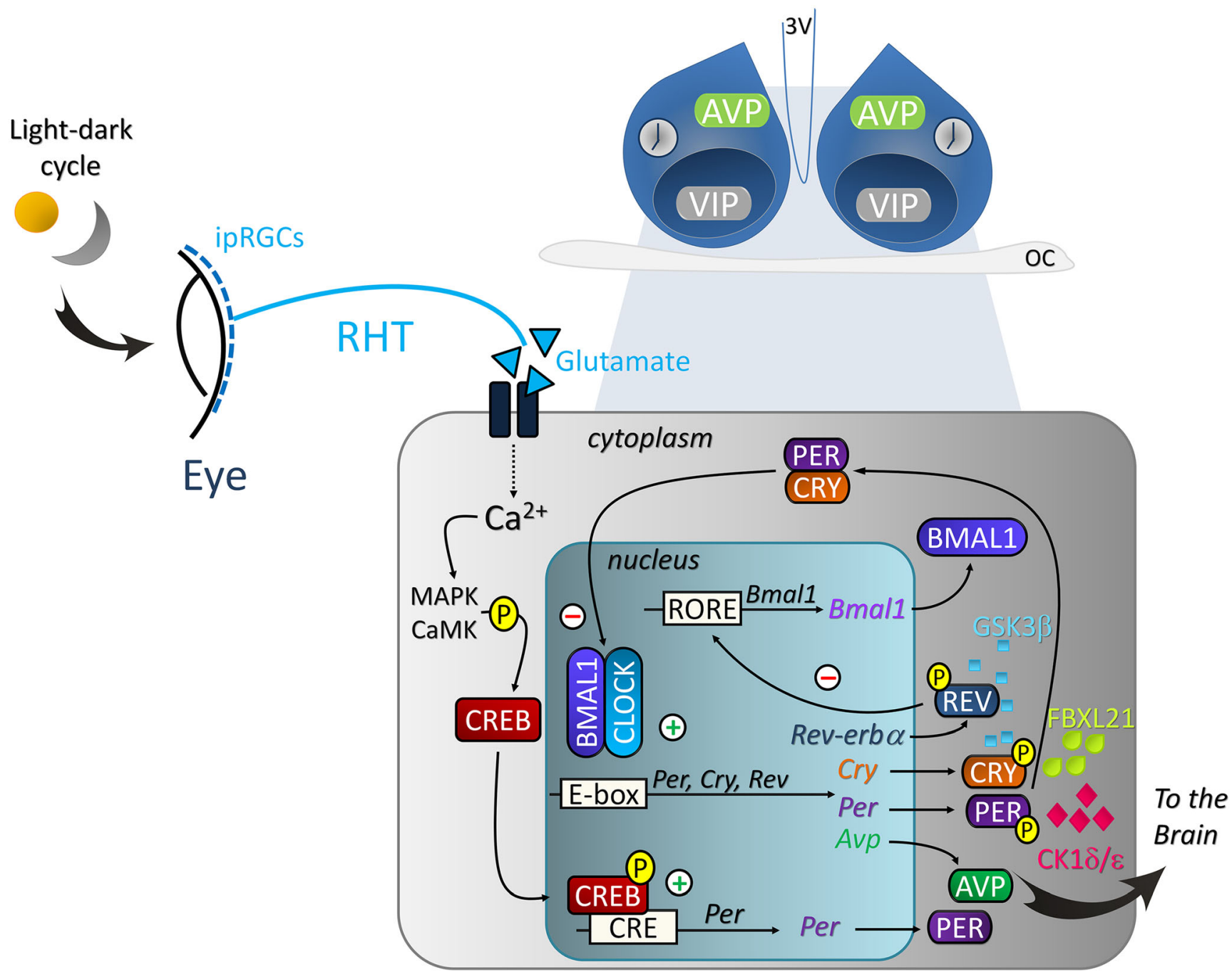

Fig. 1 The suprachiasmatic clock and the molecular code. Schematic representation of the bilateral suprachiasmatic nuclei in the hypothalamus and the anatomical subdivisions in the dorsal AVPexpressing and the ventromedial VIP-expressing regions. The SCN is principally entrained to the light-dark cycle through the RHT that connects the ipRGCs from the ganglion cell layer in the retina with the $\mathrm{SCN}$. Glutamate receptors in the SCN are activated by light signals that lead to an increase of the intracellular calcium concentration and a phosphorylation (P) of CREB (pCREB) by the activation of CaMK and MAPK kinase pathways. pCREB binds the CRE sequence in the promoter of Per genes into the nucleus to promote gene expression. Inside each SCN cell, there is a molecular pacemaker based in positive and negative feedback loops of clock gene expression. The proteins of the positive feedback loop, CLOCK and BMAL1, activate the transcription of genes Per, Cry, and Rev-Erbo via the E-box enhancers in the promoter region. The PER and CRY protein dimer complexes formed in the cytoplasm (part of the negative feedback loop) translocate into the nucleus to repress the CLOCK/BMAL1 transcriptional activity. PER and CRY protein stability and degradation in the cytoplasm are modulated by other genes such as GSK $3 \beta$, FBXL 21 and CK $1 \delta / \varepsilon$. The stability of PER-CRY proteins will determine the intrinsic period of the clock. A third loop is composed of the nuclear receptor REV-ERB $\alpha$, which represses the transcription of Bmall through binding to ROR elements (ROREs). The molecular clock in the SCN also transcribes the so-called clock-controlled genes such as vasopressin $(A v p)$ to communicate the circadian message to the rest of brain. AVP, vasopressin; $\mathrm{CaMK}, \mathrm{Ca}^{2+} /$ calmodulin-dependent kinase; $\mathrm{CK} 1 \delta \varepsilon$, casein kinase I delta, epsilon; CRE, cAMP-response element; CREB, cAMP response element-binding protein; E-box, enhancer box; FBXL, F-box and leucine-rich repeat protein; GSK $3 \beta$, glycogen synthase kinase-3 beta; ipRGCs, intrinsically photosensitive retinal ganglion cells; MAPK, mitogen-activated protein kinase; OC, optic chiasm; P, phosphoryl group; RHT, retinohyopthalamic tract; RORE, retinoic acid receptor-related orphan receptor response element; VIP, vasoactive intestinal polypeptide; $3 \mathrm{~V}$, third ventricle 

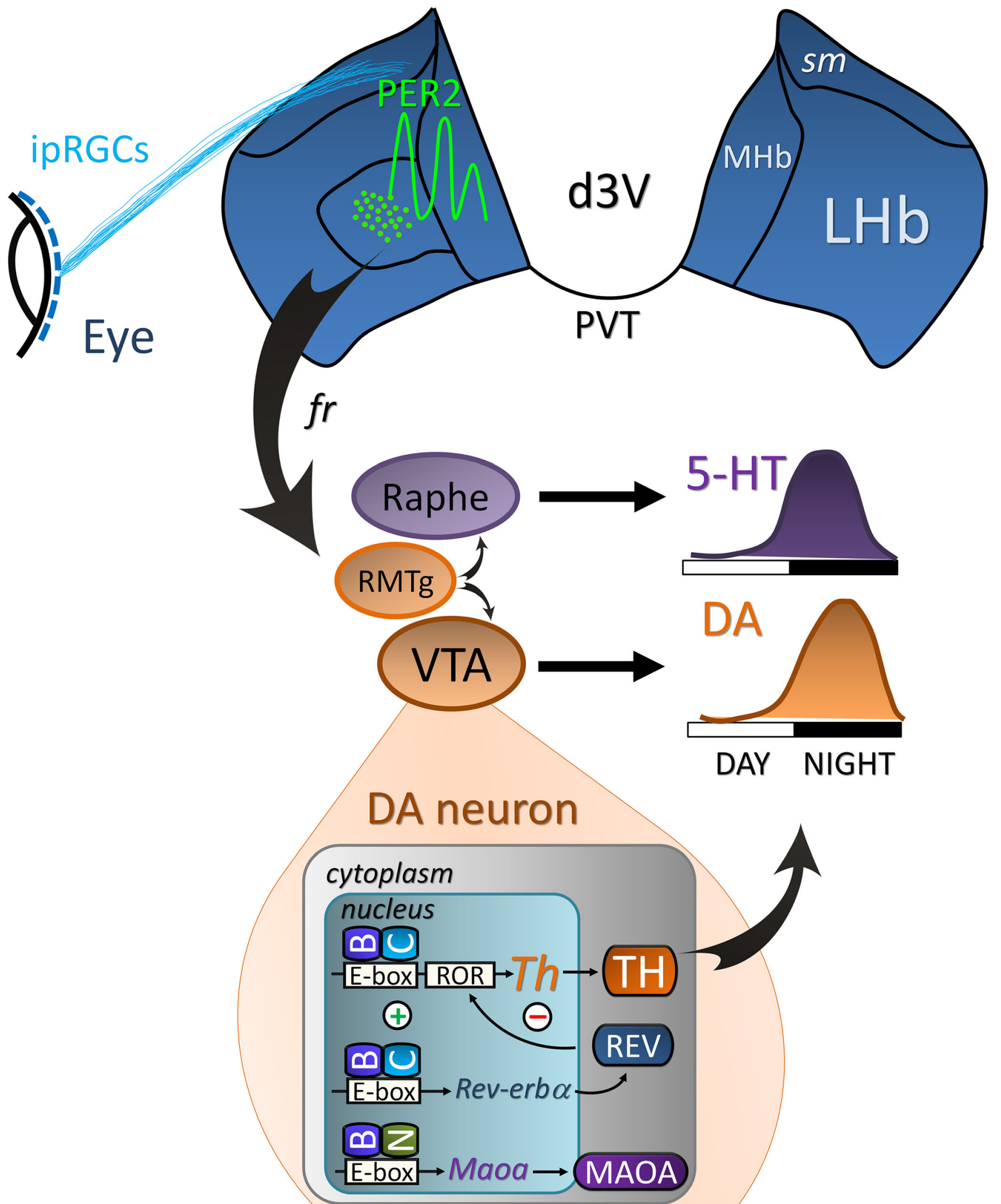
Fig. 2 The epithalamic habenula as a circadian clock. The mammalian habenula is principally subdivided into the medial $(\mathrm{MHb})$ and the lateral (LHb) parts. The LHb contains a circadian clock that expresses a rhythmically spontaneous firing rate and PER protein expression. ipRGCs in the retina project fibers to the boundary region in the LHb (the perihabenular region), suggesting that light might modulate the activity of the LHb clock. The LHb serves as a negative modulator of the monoamine system in the mid and hindbrain. LHb glutamatergic neurons project to the GABAergic RMTg, via the $f r$, which innervates the VTA and raphe nuclei for the regulation of DA and 5-HT, respectively. Importantly, DA and 5-HT release is rhythmic. In VTADA cells, the circadian clock regulates rhythms of TH, the precursor enzyme for DA synthesis and MAO-A, the principal enzyme for DA oxidation. Thus, the LHb clock might, together with the $\mathrm{SCN}$, regulate the daily rhythms of monoamines. B, BMAL1; C, CLOCK; d3V, dorsal third ventricle; DA, dopamine; E-box, enhancer box; fr, fasciculus retroflexus; ipRGCs, intrinsically photosensitive retinal ganglion cells; $\mathrm{LHb}$, lateral habenula; MAOA, monoaminoxidase $\mathrm{a}$; MHb, medial habenula; N, NPAS2; PVT, paraventricular thalamus; RMTg, rostromedial tegmental nucleus; RORE, retinoic acid receptor-related orphan receptor response element; sm, stria medularis; $\mathrm{TH}$, tyrosin hydroxylase; VTA, ventral tegmental area; 5-HT, serotonin

Publisher's note Springer Nature remains neutral with regard to jurisdictional claims in published maps and institutional affiliations. 